\title{
Retinal toxicity in long term hydroxychloroquine
}

\section{treatment}

\author{
Myron Mavrikakis, Sotirios Papazoglou, Peter P Sfikakis, George Vaiopoulos, \\ Kostantinos Rougas
}

\begin{abstract}
Objective-To report clinical experience from patients with rheumatoid arthritis (RA) and systemic lupus erythematosus (SLE) who were receiving recommended doses of hydroxychloroquine for more than six years, and were monitored for evidence of hydroxychloroquine related retinopathy every six months.

Methods-A prospective (and continuing) evaluation was made of the potential retinal toxicity of hydroxychloroquine in a cohort of 360 Greek patients followed for RA and SLE, 58 of whom have received long term treatment ( $>$ six years). Fundoscopy, colour vision tests, dark adaptation tests, visual field testing, automated perimetry, and electroretinogram were performed every six months.
\end{abstract}

Results-Among 58 patients receiving hydroxychloroquine for more than six years, two relatively young women $(3.5 \%)$, one treated for RA and the other treated for SLE, developed characteristic hydroxychloroquine related toxic retinal lesions after cumulative doses of $700 \mathrm{~g}$ (6.5 years) and $730 \mathrm{~g}$ (8 years) of hydroxychloroquine, respectively. Bilateral visual acuity was $6 / 6$ and $6 / 7 \cdot 5$, respectively; both patients had normal colour perception. Despite an early diagnosis and cessation of treatment, permanent visual field paracentral scotomata in both patients, and persisting lesions in fluorescein angiography in the patient with SLE, were observed at 4.5 and 3 years of follow up, respectively. No other specific cases of hydroxychloroquine related retinopathy have to date been identified in the remaining 302 patients.

Conclusion-Cases of irreversible, hydroxychloroquine related retinopathy in patients who did not receive overdoses have not been reported previously. The present observations in two relatively young patients should raise our concern regarding the long term usage of an increasingly popular medication in rheumatology practice.

(Ann Rheum Dis 1996; 55: 187-189)

Almost $50 \%$ of patients prescribed any of the slow acting antirheumatic drugs (SAARDs) discontinue their treatment within two years because of inefficacy or toxicity. Compared with the other SAARDs, the antimalarial agents have a major advantage in their lack of serious adverse effects such as bone marrow suppression, promotion of opportunistic infections, or malignancy. ${ }^{1}$ Their well established beneficial effect in rheumatoid arthritis (RA), systemic lupus erythematosus (SLE), and Sjögren's syndrome, together with their antithrombotic and lipid decreasing action and lack of life threatening toxicity are likely to lead to a continuing increase in the number of patients treated with these drugs. ${ }^{1-3}$ The recently proposed mechanism of immunological action of antimalarial agents, namely interference with normal antigen presentation by increasing the intracytoplasmic $\mathrm{pH},{ }^{4}$ provides an exciting basis for the usefulness of these agents. However, because of the well known ocular toxicity of antimalarial agents, some rheumatologists underutilise them, while fear of sustaining damage to their eyes deters many patients from taking them. According to the information leaflet that currently accompanies hydroxychloroquine medication, the practitioner prescribing the drug should expect to receive an assessment report from the patient's ophthalmologist every three months.

The present study was begun in 1985 and is a continuing longitudinal evaluation of the potential retinal toxicity of hydroxychloroquine in Greek patients suffering from RA and SLE (S Papazoglou et al: preliminary findings presented at the XVIIth International League of Associations for Rheumatism Congress of Rheumatology, Rio, Brazil, 1989). From a total of 360 patients included in the study up to May 1995, the clinical experience from a subgroup of 58 patients receiving the drug in recommended doses for more than six years is described here.

\section{Patients and methods}

STUDY POPULATION

This prospective study included consecutive patients with RA or SLE, followed at the Departments of Clinical Therapeutics and Internal Medicine of the Athens University Medical School and the Rheumatology Department of the General Hospital, Athens, Greece, who were being treated with hydroxychloroquine. Abnormality of visual function or retinal appearance at baseline were the only exclusion criteria. Of 360 patients that have been studied to date, the subgroup who have received long term treatment comprised 58 


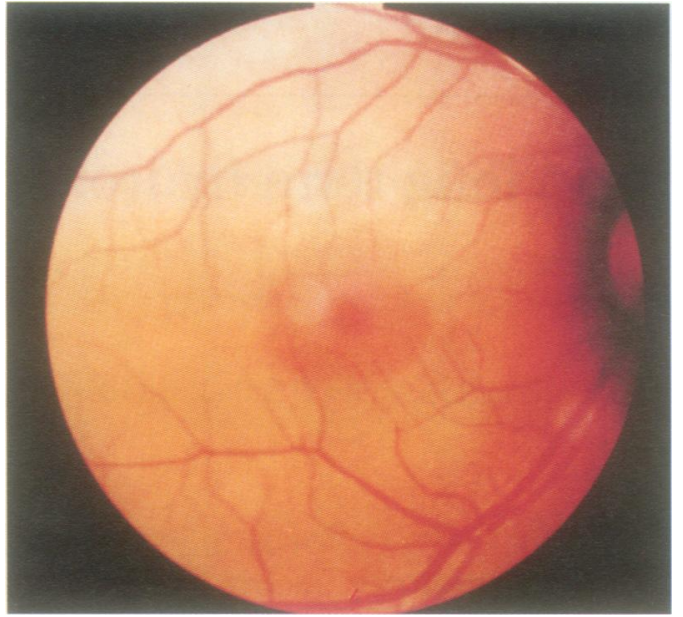

Figure 1 Fundus photograph of the right eye of a 39 year old patient with rheumatoid arthritis, treated with recommended doses of hydroxychloroquine for 6.5 years, showing antimalarial associated retinopathy with the characteristic 'bull's eye' appearance, resulting from localisation of the pigment in a ring pattern around the fovea.

patients (47 women and 11 men), 39 with RA and 19 with SLE, with a mean duration of treatment of 7.5 years (range 6-11 years). Hydroxychloroquine doses administered to these patients were less than $6.5 \mathrm{mg} / \mathrm{kg} /$ day and none had impairment of either liver or renal function. Normal glucose-6-phosphate dehydrogenase concentrations were recorded before initiation of treatment in all patients.

\section{MONITORING}

All ophthalmological evaluations were performed by one of the authors (K R), every six months. In addition to routine and fundoscopic testing, a detailed colour vision test (15 Hue Panel Test), dark adaptation tests, visual field testing, automated perimetry tests, and electroretinogram (scotopic, photopic, and 50 $\mathrm{Hz}$ flickers) were performed. Fluorescent angiography was also performed in patients presenting evidence of fundoscopic lesions.
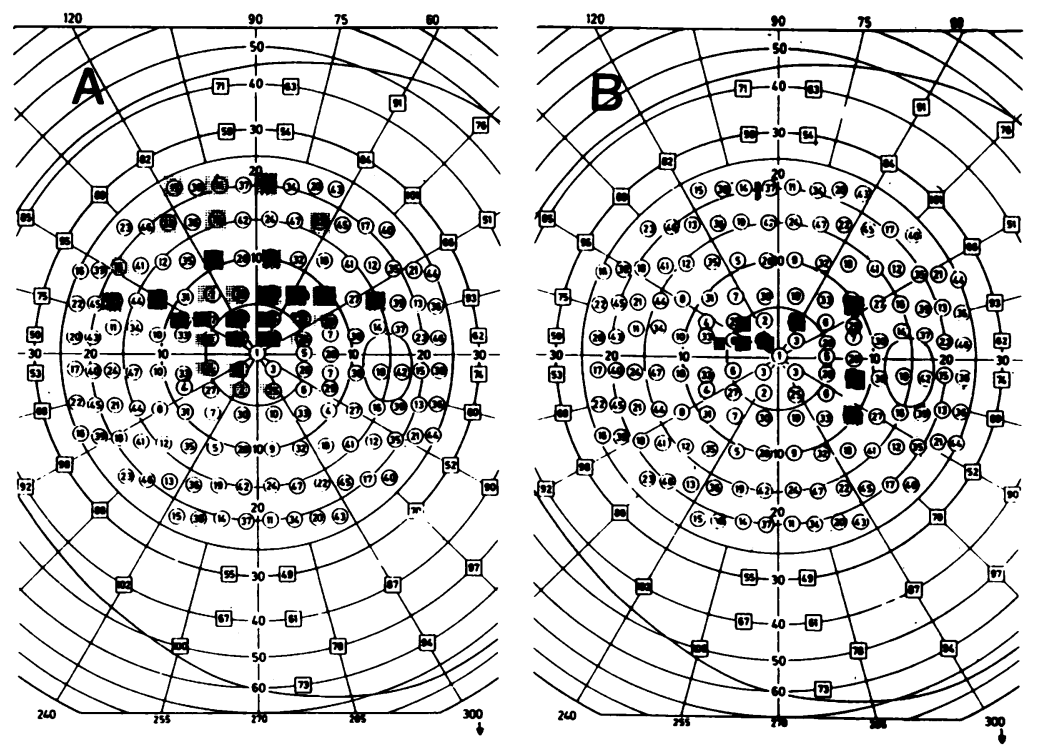

Figure 2 Paracentral scotomata, as shown on visual field plot of the right eye of a 39 year old patient with rheumatoid arthritis treated with recommended doses of hydroxychloroquine for 6.5 years. A: At cessation of treatment; B: $4 \cdot 5$ years after cessation of treatment.

\section{Results}

LONG TERM TREATMENT

Among the 58 patients treated long term, two middle aged women whose cumulative dosages of hydroxychloroquine were at the top end of the range of cumulative dosage of the whole group developed toxic, irreversible retinopathy lesions that were clearly attributable to hydroxychloroquine.

Patient 1-A 39 year old woman with RA, whose weight varied in the range $56-62 \mathrm{~kg}$ over the years of treatment, developed characteristic retinal changes after 6.5 years of treatment with hydroxychloroquine (400 $\mathrm{mg} /$ day for three years, then $200 \mathrm{mg} /$ day for 3.5 years, approximately; cumulative dose approximately $700 \mathrm{~g})$. Bilateral best corrected visual acuity (6/6) and colour perception were normal, but fundoscopic lesions (fig 1) and paracentral scotomata (fig 2A) in the right eye were observed. These lesions were also demonstrated in fluorescein angiography with the beginnings of a mask defect on the macula; the respective electroretinogram was suppressed. Comorbidities that could have caused these lesions were not identified.

Despite early diagnosis, cessation of treatment, and attempts to bind hydroxychloroquine with ammonium chloride or dimercaprol, the condition of this patient remained relatively stable, and though a recent fluorangiogram appeared normal, unilateral paracentral scotomata were evident in the visual field (fig 2B) in 4.5 years of follow up, while the respective electroretinogram was also suppressed, indicating the presence of retinopathy.

Patient 2-A 58 year old woman with SLE, weighing $69 \mathrm{~kg}$, developed retinal changes after eight years of treatment with hydroxychloroquine $(400 \mathrm{mg} /$ day for two years, then $200 \mathrm{mg} /$ day for six years; cumulative dose $730 \mathrm{~g}$ ). Colour perception was normal, but a slight bilateral decrease in best corrected visual acuity $(6 / 7 \cdot 5)$ was observed. Paracentral scotomata on both optical fields, fundoscopic lesions of a characteristic appearance that were similar to the lesions depicted in figure 1 , abnormal fluorescein angiography, and suppressed retinograms were also observed.

This second patient's condition, in common with that of the first, did not change during follow up: bilateral visual field tests remained abnormal, the amplitude of the B wave on the retinogram was markedly reduced, and characteristic hydroxychloroquine related retinal changes were evident in fluorescein angiography three years after cessation of treatment (fig 3).

PATIENTS TREATED FOR LESS THAN SIX YEARS We have not observed any case of hydroxychloroquine related retinal toxicity in the remaining 302 patients of the study population who, at the time of this study, had received the drug in recommended doses for less than six years (mean cumulative dose of $255 \mathrm{~g}$ ). SLE related changes, ${ }^{5}$ or non-specific changes such as senile retinal degeneration, cataracts, 

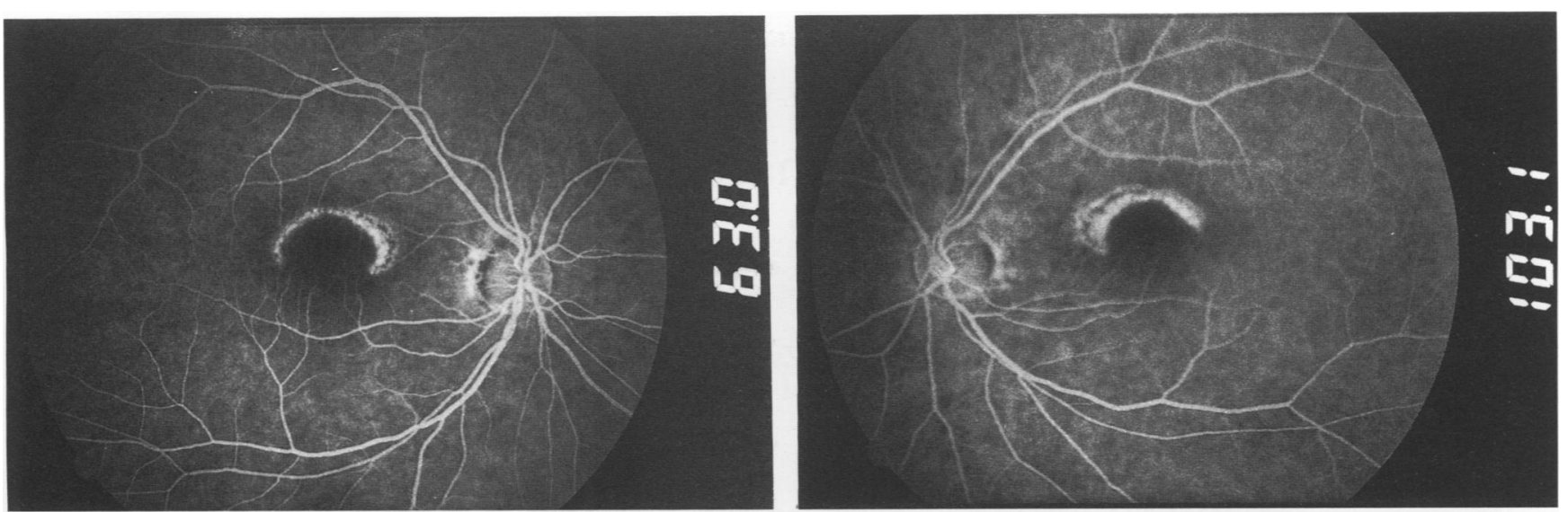

Figure 3 Bilateral lesions in fluorescein angiography of hydroxychloroquine related retinopathy persisting three years after cessation of treatment in a patient with systemic lupus erythematosus who had been treated with recommended doses of hydroxychloroquine for eight years.

myopic degeneration, chiasmatic syndrome, pigmented retinopathy, and Eales' disease were documented in 10 patients at some time during the frequent evaluations, but these changes were not attributable to hydroxychloroquine.

\section{Discussion}

Retinal toxicity was recognised as the main side effect of antimalarial agents in 1957, and since then more than 300 cases have been reported in the literature. ${ }^{1}$ The vast majority of these cases were related to chloroquine treatment, probably reflecting the relative use of chloroquine and the other antimalarial agents. ${ }^{6}$ Bernstein analysed thoroughly all cases designated as hydroxychloroquine induced retinopathy that were reported in the literature between 1960 and 1989 , or reported to the Food and Drug Administration from 1975 to 1990. He found that 20 cases fulfilled validated criteria, ${ }^{78}$ but considered that only two of the 20 represented true hydroxychloroquine related retinopathy that was attributable to daily doses not exceeding the recommended dose of $6.5 \mathrm{mg} / \mathrm{kg}$, in the absence of renal insufficiency. However, these two patients had taken the drug for 14 and 10 years, respectively. ${ }^{7}$

Two additional cases of hydroxychloroquine related retinopathy were reported by Weiner et $a l ;{ }^{9}$ one had received excessive dosage $(6 \cdot 1-12 \cdot 2 \mathrm{mg} / \mathrm{kg} /$ day for 10 years $)$, and the other received a dose of $6 \cdot 1 \mathrm{mg} / \mathrm{kg} /$ day over a 20 year period, with a cumulative dose of $2920 \mathrm{~g}$. Whether the cumulative dose, rather than a greater daily dose, is important in determining overdosage, toxicity, or both, remains controversial..$^{710}$ Nevertheless, according to Wallace, ${ }^{1}$ and to the best of our own knowledge, irreversible retinal changes in patients with normal renal function, taking hydroxychloroquine in recommended doses for less than 10 years, have not been reported previously.

The two cases of hydroxychloroquine retinopathy among 58 long term treated patients (3.5\%) represent a substantial prevalence that is clearly greater than that reported in other studies. ${ }^{7-13}$ The difficulty in avoiding exposure to the sun in Greece may represent an additional risk factor ${ }^{1112}$ that contributed to the retinal toxic effect of hydroxychloroquine in our patients. At present, it remains unclear why such cases have not been reported in other areas with a particularly sunny climate. Unknown factors, including unusual enzymatic deficiencies analogous to glucose-6-phosphate dehydrogenase deficiency, may predispose Greek patients to toxicity.

Our finding of no cases of retinal toxicity in our patients during the first six years of hydroxychloroquine treatment is in accordance with other reports, ${ }^{71013}$ suggesting that frequent and meticulous ophthalmological assessment of patients receiving hydroxychloroquine is not neccessary during the first years of treatment. However, we disagree with Bernstein's conclusion that no risk of retinopathy exists during the first 10 years of hydroxychloroquine treatment in recommended doses. ${ }^{7}{ }^{8}$ In view of the irreversible cases of retinopathy reported above, prolonged use of this drug should be accompanied by mandatory ophthalmological evaluation at least two or three times a year, even if the daily dose is maintained at less than $6.5 \mathrm{mg} / \mathrm{kg}$.

We thank Dr Sasa Alimisi for helpful comments.

1 Wallace D J. Antimalarial therapies. In: Wallace D J, Hahn B H, eds. Dubois' Lupus Erythematosus, 4th edn. Hahn B H, eds. Dubois' Lupus Erythematosus

2 Felson D T, Anderson J J, Meenan R F. The comparative efficacy and toxicity of second-line drugs in rheumatoid arthritis. Results of two metaanalyses. Arthritis Rheum 1990; 33: 1449-61.

3 Wallace D J. Antimalarial agents and lupus. Rheum Dis Clin N Am 1994; 20: 243-64.

4 Fox R I. Mechanism of action of hydroxychloroquine as an antirheumatic drug. Semin Arthritis Rheum 1993; 23: 82-91.

5 Stafford-Brady F J, Urowitz M B, Gladman D D, Easterbrook M. Lupus retinopathy. Arthritis Rheum 1988; 31: 1105-10

6 Finbloom D, Silver K, Newsome E, Gunkel R. Comparison of hydroxychloroquine and chloroquine use in the development of retinal toxicity. $\mathcal{F}$ Rheumatol 1985; 12: 692-4

7 Bernstein H N. Ocular safety of hydroxychloroquine. Ann Opthalmol 1991; 23: 292-6.

8 Bernstein $\mathrm{H} \mathrm{N}$. Ocular safety of hydroxychloroquine sulfate. South Med F 1992; 85: 274-8.

9 Weiner A, Sandberg M A, Gaudio A R, Kini M M, Berson E L. Hydroxychloroquine retinopathy. $A m \mathcal{f}$ Opthalmol 1991; 112: 528-34.

10 Easterbrook $M$. The ocular safety of hydroxychloroquine. Semin Arthritis Rheum 1993; 23: 62-7.

11 Mackenzie A H. Dose refinements in long-term therapy of rheumatoid arthritis antimalarials. Am $\mathcal{F}$ Med 1983; 18: $40-5$.

12 Lanham J G, Hughes G R V. Antimalarial therapy in SLE. Clin Rheum Dis 1982; 8: 279-98.

13 Morsman C D, Livesey S J, Richards I M, Jessop J D, Mills P V. Screening for hydroxychloroquine retinal toxicity: is it necessary? Eye 1990; 4: 572-6. 